\title{
Towards Establishing Compact Imaging Spectrometer Standards
}

\author{
E Terrence Slonecker ${ }^{1 *}$, David W Allen ${ }^{2}$ and Ronald G Resmini ${ }^{3}$
}

\author{
${ }^{1}$ U.S. Geological Survey, Eastern Geographic Science Center, 12201 Sunrise Valley Drive, Reston, Virginia 20192, \\ USA
}

${ }^{2}$ National Institute of Standards and Technology, Sensor Sciences Division, 100 Bureau Drive, Gaithersburg, Maryland 20899, USA

${ }^{3}$ The MITRE Corporation, 7594 Colshire Drive, MS T630, McLean, Virginia 22102, USA

\begin{abstract}
Remote sensing science is currently undergoing a tremendous expansion in the area of hyperspectral imaging (HSI) technology. Spurred largely by the explosive growth of Unmanned Aerial Vehicles (UAV), sometimes called Unmanned Aircraft Systems (UAS), or drones, HSI capabilities that once required access to one of only a handful of very specialized and expensive sensor systems are now miniaturized and widely available commercially. Small compact imaging spectrometers (CIS) now on the market offer a number of hyperspectral imaging capabilities in terms of spectral range and sampling. The potential uses of $\mathrm{HSI} / \mathrm{CIS}$ on UAVs/UASs seem limitless. However, the rapid expansion of unmanned aircraft and small hyperspectral sensor capabilities has created a number of questions related to technological, legal, and operational capabilities. Lightweight sensor systems suitable for UAV platforms are being advertised in the trade literature at an ever-expanding rate with no standardization of system performance specifications or terms of reference. To address this issue, both the U.S. Geological Survey and the National Institute of Standards and Technology are developing draft standards to meet these issues. This paper presents the outline of a combined USGS/NIST cooperative strategy to develop and test a characterization methodology to meet the needs of a new and expanding UAV/CIS/HSI user community.
\end{abstract}

\section{Keywords}

Hyperspectral imaging, Compact imaging spectrometers, Unmanned Aerial Vehicles (UAVs), Unmanned Aircraft Systems (UAS), Drones, Imaging spectroscopy

\section{Introduction}

Remote sensing technology is currently undergoing a rapid technological expansion in the area of Unmanned Aerial Vehicles (UAVs), sometimes referred to as Unmanned Aircraft Systems (UAS), drones, or remotely piloted aircraft (RPA). From complex attack aircraft, such as the Predator, developed by the Department of Defense and currently an integral part of battlefield strategy, to hand held, model-airplane-like light weight systems that are launched by hand, UAVs span a wide range of platforms and capabilities for which the only common feature is that they fly without an on-board pilot.

The rapid expansion of both unmanned aircraft and sensor capabilities has created a number of questions related to technological and operational capabilities (and constraints) of sensors on unmanned aerial platforms and on the legality of imaging during over-flights [1]. Lightweight sensor systems suitable for UAV platforms are being advertised in the trade literature at an ever-increasing rate and with no evident standardization of systems requirements or specifications.

The rapidly expanding field of hyperspectral imaging (HSI), also known as imaging spectroscopy, has also produced relatively lowcost and light-weight sensor packages for UAVs. HSI systems, as applied to UAV technology, separate electromagnetic (EM) energy (light) into multiple bands of imagery, each band recording reflected spectral radiance generally in the $400 \mathrm{~nm}$ to $2500 \mathrm{~nm}$ spectral range, also known as the "solar reflected" portion of the electromagnetic (EM) spectrum. The result is the collection of a stack of co-registered images, often referred to as an "image cube" that can be analyzed using the same spectroscopic methods used in analytical chemistry.

The spectral and radiometric calibration and the spatial/imageforming capability of HSI sensors, and their presentation to potential end-users and consumers thus deserve particular emphasis. HSI

*Corresponding author: E. Terrence Slonecker, U.S. Geological Survey, Eastern Geographic Science Center, 12201 Sunrise Valley Drive, Reston, Virginia 20192, USA, Tel: +1-703-648-4289, Fax: +1-703-648-4603, E-mail: tslonecker@usgs.gov

Received: January 28, 2016: Accepted: March 28, 2016: Published: March 31, 2016

Copyright: (C) 2016 Slonecker ET, et al. This is an open-access article distributed under the terms of the Creative Commons Attribution License, which permits unrestricted use, distribution, and reproduction in any medium, provided the original author and source are credited. 
sensors-regardless of whether they are ground-based, airborne, space borne, or used on the lab bench attached to microscopes, measure spectral radiance as a function of wavelength. And they do so regardless of where they are designed to sense within the electrooptical (EO) wavelength range from the ultraviolet $(\sim 0.25 \mu \mathrm{m})$ to the long wave infrared $(\sim 14.0 \mu \mathrm{m})$. Wavelength (or spectral) calibration (usually achieved in the laboratory with $\mathrm{Hg}$ discharge lamps, laser or monochromator sources) precedes radiometric calibration. Though the reflectance spectrum is a key information-carrying datum in any spectral sensor, it is derived from the radiance data and as such, radiometric calibration and standards for it are essential. Attention should also be paid to radiometric characterization even for those sensors to which some sort of flat-fielding or ratioing with a uniform reflectance target is applied.

A review of the radiometric and reflectance quantities and definitions is provided by Schaepman-Strub et al. (2006) [2]. In that paper, consistent uses of common terms are examined and more precise usage that is based on physical quantities is advocated. Herein, the focus is on HSI sensors that passively record spectral radiance and are suitable for operation on small UAVs. Many current HSI sensors record spectral radiance in units of micro-watts per square centimeter per steradian per micrometer, $\mu \mathrm{W} / \mathrm{cm}^{2} \cdot \mathrm{sr} \cdot \mu \mathrm{m}$, but other spectral radiance units are in use. Spatial/image-forming characterization will also be considered.

This paper addresses the issue of the need for standards for CIS. This is also the objective of a combined and cooperative strategy being developed by the National Institute for Standards and Technology (NIST) and the United States Geological Survey (USGS) to develop standards to help meet the needs of a new and expanding UAV/ hyperspectral user community.

\section{Background}

Compact imaging spectrometers (also referred to as hyperspectral imagers) are being used for a growing number of applications outside of the military arena. The lower cost and size (on the order of several $\mathrm{kg}$ or less) has opened up a range of new applications. Some examples include precision agriculture [3], medical diagnostics [46], sorting recyclables $[7,8]$, food and pharmaceutical quality control [9-11], forensic medicine [12-14], the detection of environmental contaminates [15], detecting biological contaminants [16], detecting invasive species [17-19], and many others. Their compact size has allowed their use on bench-tops, observing conveyor belts in factories, and ground-based and airborne platforms (including Unmanned Aerial Vehicles). While the market for compact hyperspectral imagers is growing rapidly, performance metrics and validation methods have not kept pace. There is a growing level of interest in the definition, classification and reporting of hyperspectral sensor specifications and standards, especially those related to compact imaging spectrometers and the UAV market in general. To address the needs of this emerging market and user community, a workshop was held on April 22, 2015, at the SPIE (International Society for Photonics and Optics) DSS Sensing and Technology Conference in Baltimore, Maryland. The workshop featured speakers from NIST, USGS, the MITRE Corporation and SpecTIR, an established hyperspectral remote sensing firm. The goal of the workshop was to discuss what is the range of standards related to-and that should be applied to HSI, what standards that are currently available (and in use), and on which areas standards development should focus on. Several issues were identified based on input from the participants:

- The need for standard terminology/terms of reference

- The need for sensor characterization (including calibration)

- A definition of methods and best practices (collection through processing)

\section{- A set of standard spectral signatures}

\section{- A set of standard hyperspectral processing algorithms}

It was noted that given the broad range of applications, there will likely be a need for application specific standards, methods, and best practices. The task of addressing the full range of these topics falls on the community in coordination with standards development organizations. Sensor characterization is one area for the central focus of the NIST/USGS collaboration. Sensor characterization includes using a set of tests that help to understand the sensor performance. This process generally includes the use of calibration standards. Calibration and validation of the performance of these systems allow for applications that make use of these data to collect values of known quantities through traceability to national standards. Calibrated and characterized hyperspectral imagers in combination with best practices will facilitate the transition from qualitative efforts to quantitative, robust, and repeatable results.

The National Institute of Standards and Technology (NIST) is the United States government agency responsible for developing, maintaining, and disseminating national standards-realizations of the International System of Units (SI). A key component related to measurement standards is the concept of measurement uncertainty. Traceability to NIST implies an unbroken chain of measurements with expressed uncertainties for the measurement values related to a standard.

NIST is also responsible for assessing the measurement uncertainties associated with the values assigned to these measurement standards. Traceability to SI units requires the establishment of an unbroken chain of calibrations to specified references [20]. NIST serves as the United States' national metrology institute (NMI). NIST works closely with NMIs in other countries to ensure mutually agreement of measurement scales of common interest. Agreement on international scales fosters mutual recognition and acceptance of equivalence. NIST has a long history of providing standards related services to the remote sensing community. Most of that work has supported the pre-launch calibration to Earth observing satellite sensors. These activities either direct calibration of sensors or through the transfer of scales. Many of the same standards and calibration facilities can be leveraged to serve the emerging CIS market, either directly or indirectly.

USGS has long provided the calibration service for analog mapping cameras. The Optical Sciences Laboratory provides a calibration service that includes: focal length, calibrated fiducials, lens distortion characteristics, and other parameters [21]. However, because of the changing nature of overhead mapping technology, USGS announced in 2011 that it would not develop or put forth a digital mapping camera certification requirement but would still plan a course-of-action to maintain expertise and to add value to the digital remote sensing technologies used in Federal agencies, government at all levels, and industry [22].

In support of the evaluation of remote sensing technologies, the USGS has established USGS National Test Ranges in South Dakota, Missouri, Colorado, with additional ranges planned elsewhere [23]. These ranges are designed to facilitate geometric evaluation of remote sensing technologies from visible to infrared electro-optical aerial and satellite sensors. The ranges can also be enhanced to support evaluation of geospatial and radiometric comparison of sensors, and to evaluate additional remote sensing technologies such as light detection and ranging (LiDAR) and synthetic-aperture radar (SAR).

The intent of this proposed characterization service is to serve customer needs at a suitable level. The basic radiometric and spectral characterization service will provide values of spectral radiance responsivity that will allow the end user to acquire quantitative data with associated uncertainties that are traceable to NIST. The 
advanced level of characterization will be tailored to the customers' requirements and takes into consideration the intended application. This second level is in addition to the basic characterization service in which spectral signatures and hyperspectral scenes are used as standards. These can be provided by the customer or by NIST. These services are primarily intended to provide sufficient information to gauge the level of confidence of the spectral performance of the CIS. The spatial imaging performance as it relates to spatial accuracy of digital cameras will heavily leverage the work that has been conducted by the USGS Remote Sensing Technologies program. See Sections 5.2 and 5.4 below.

\section{Characterization}

\section{Compact imaging spectrometer description}

One standard practice being proposed here is that each compact imaging spectrometer should be described by a standard set of specifications with a minimum set of information elements. These may include: spectral wavelength range, spectral resolution, spectral band width, focal plane array characteristics (e.g., dimensions), sensor type (push-broom, whisk-broom, framing), digital interface type, bit depth, data storage capacity, size, weight, and power requirements, standard mounting bracket, environmental operating range, and any additional products that may not be included as part of the standard product (e.g., software). This information will facilitate the selection in the procurement process and help in matching the right sensor to a given application.

\section{Radiometric and spectral characterization}

Fundamental to imaging spectrometers is the spectral radiance responsivity. Radiometric calibration provides radiance responsivity for each pixel in the focal plane array of the imaging spectrometer. The radiance responsivity allows for the signal output of the imaging spectrometer to be related to the physical units of radiance, allowing the instrument to be used in a quantitative manner relative to an absolute scale. This practice has been well described in [24-27]. The calibration results can also provide assurance of basic performance to assist in validating specifications. Radiometric calibration is preceded by spectral calibration which also follows existing, standard bestpractices. Spectral (or wavelength) calibration is usually achieved in the laboratory with $\mathrm{Hg}$ discharge lamps, laser or monochromator sources. In some applications, calibration may also be achieved with onboard light sources but this generally drives up size, weight, and power (SWaP) requirements.

The national scale of radiance originates from the NIST Primary Optical Watt Radiometer (POWR) [28] and is transferred via the Spectral Irradiance and Radiance Calibrations Using Uniform Sources (SIRCUS) facility [29] and is disseminated using transfer radiometers. The SIRCUS facility is based on a collection of tunable lasers that provide high power with narrow spectral bandwidths. Numerous image frames are used to compose the spectral response of each spectral band. The uncertainties provided by SIRCUS are on the order of $0.1 \%$ at $\mathrm{k}=2$, where $\mathrm{k}$ is the coverage factor and $\mathrm{k}=2$ represents a $95 \%$ confidence internal, assuming a normal distribution. (http://physics.nist.gov/cuu/Uncertainty/coverage.html).

This facility provides very high resolution spectral radiance needed to fully characterize the band response of the spectrometer. Spectrally broadband sources based on lamp illuminated integrating spheres provide the radiance scale. These broadband sources allow the determination of radiance responsivity over the full spectral range over one image frame. The nominal uncertainties for these sources is $0.5 \%$ in the visible to several percent outside of the visible, at $\mathrm{k}=$ 2. These spatial uniform sources provide the radiance responsivity for each of the spatial pixels allowing for flat fielding of the response function. For each wavelength band and focal plane array pixel element, the appropriate measurement equation 1 is

$$
L=\frac{s-s_{d}}{t \cdot R}
$$

Where $L\left[\mu \mathrm{W} / \mathrm{cm}^{2} \cdot \mathrm{sr} \cdot \mu \mathrm{m}\right]$ is the spectral radiance, $S$ [counts] is the signal (or digital number, $\mathrm{DN}$ ) for the pixel, $S_{\mathrm{d}}$ [counts] is the dark signal DN, $t[\mathrm{~s}]$ is the exposure time, and $R\left[(\right.$ counts $/ \mathrm{s})\left(\left(\mathrm{cm}^{2} \cdot \mathrm{sr} \cdot \mu \mathrm{m}\right) /\right.$ $\mu \mathrm{W})]$ is the spectral radiance responsivity. The exposure time $t$ enters into the measurement equation because the counts read from each pixel element (or focal plane array detector) are proportional to the number of photons absorbed by that element, and hence to the time during which photons are allowed to impinge upon the array. This is commonly known as dwell time. The spatial performance will be evaluated using methods described in references [30,31], and which are based on measuring the spatial modulation transfer function (MTF). For small CIS applied to targets that are close to the aperture (e.g., spectral microscopy) and/or for which the acquisition of highresolution imagery is a collection requirement (CIS on UAVs), the use of resolution of charts (and perhaps the design of new charts specifically for spectral imaging systems) is required. The utilization of key aspects of the USGS's existing protocol for the characterization of the image forming capability of digital sensors is discussed below in sec. 5.4. Also, an excellent discussion of both HSI spectral error (smile) and spatial error (keystone) can be found in Dadon et al. [32].

Reported measurement values: The values reported for the imaging spectrometer primary characterization performance includes, radiance responsivity, and derived values including signalto-noise ratio (SNR), straylight, linearity, wavelength accuracy, and spatial characterization/modulation transfer function (MTF). These values will be accompanied with a description of the measurement method, equation, and estimated measurement uncertainties.

\section{Advanced characterization}

Advanced characterization offered by NIST, is based on the use of the NIST Hyperspectral Image Projector (HIP) [33,34]. Since spectral imagers are tasked with measuring complex spatial and spectral scenes, it is advantageous to use test standards that mimic the spectral and spatial complexity of reality. Compact imaging spectrometers are designed to take images composed of many spectral bands, not just the minimum three components used by

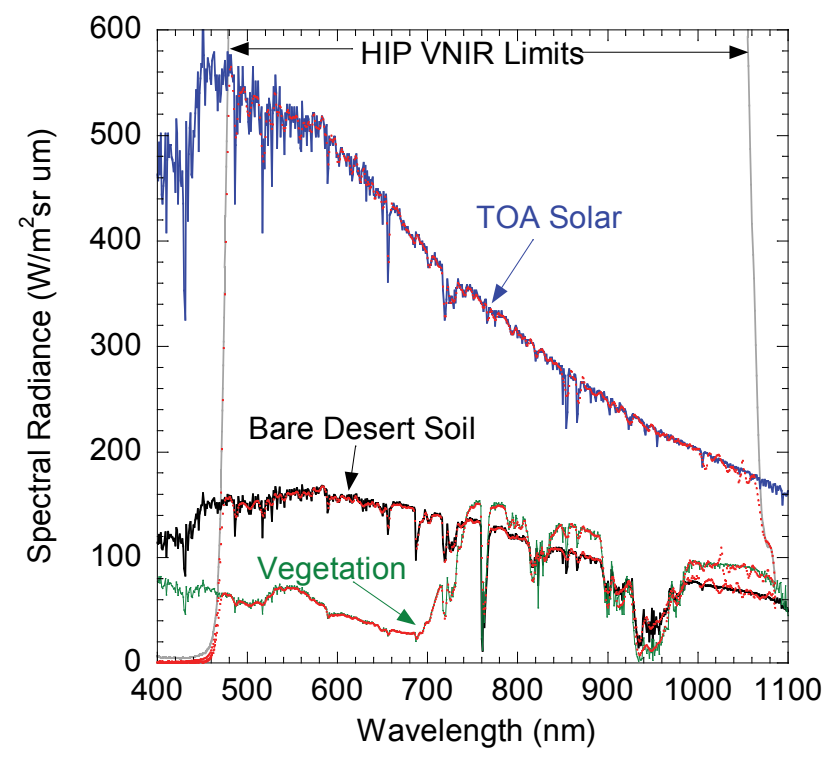

Figure 1: Sample spectra produced with the NIST HIP. The plot provides the original measured spectra and the matched spectra in red (overlaid), solar radiance, soil, and vegetation. This type of test will permit the identification of spectral flaws and limits from test CIS systems (From http://www.nist.gov/ pml/div685/grp04/hip.cfm/ ). 


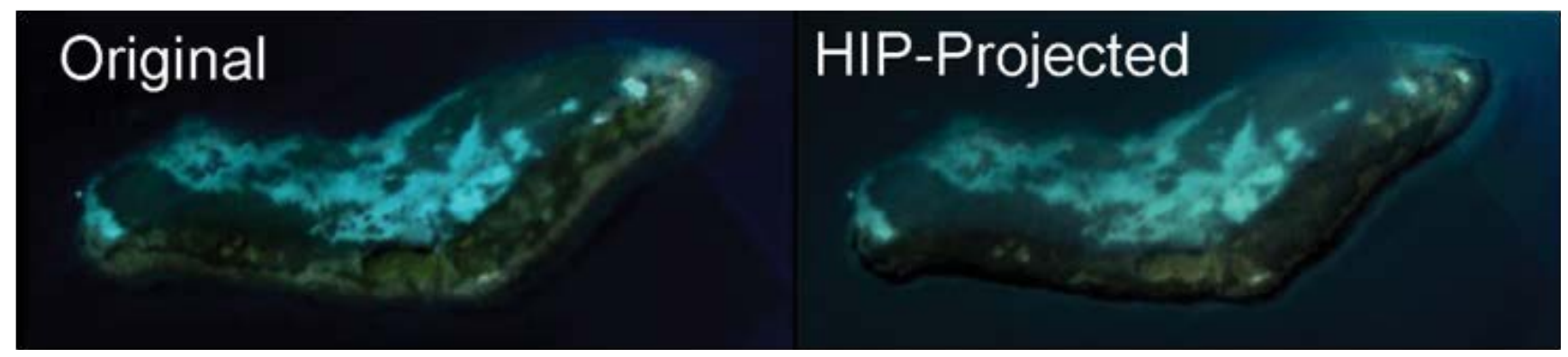

Figure 2: This image is a color composite from an airborne hyperspectral datacube showing a coral reef. The hyperspectral scene was matched, projected and collected with atest imaging spectrometer. This process is beingviewed as an important test protocol for CIS systems. From Allen et al. 2009 [35]

common digital cameras (i.e., red, green and blue). These images are referred to as hyperspectral because each pixel contains information for hundreds or thousands of narrow spectral bands. The purpose of the (HIP) is to enable the projection of hyperspectral images into sensors, simulating realistic scenes both spectrally and spatially, for performance testing and evaluation of the sensor instruments in the laboratory. For validation, the HIP provides a significant advantage over the primary calibration alone. Evaluating imaging spectrometers poses a challenge in that the spectral response function (SRF) and the point spread function (PSF) are not easily separated from each other [30]. The HIP can provide scenes with full spectral and spatial content that mimics reality [34-37]. Thus, the evaluated performance using the HIP can be expected to match what would be encountered in real world conditions. Alternatively, the hyperspectral test scene generated can also be synthetically generated [38]. Furthermore, the spectra and scene selected can be collected from a variety of sources in order to match the specific application [39,40]. Using scenes generated from application specific data as a validation standard provides the best insight in performance that can be acquired inside the laboratory while minimizing the variability and costs associated from field evaluations. Since the data used to generate the projected scene is digital in nature, it can readily be disseminated to other laboratories that may use similar technology. In that case, the hyperspectral data cube for any given application can be used as a standard reference. Figure 1 shows an example of spectra matched by the HIP while Figure 2 shows a color composite from a hyperspectral data cube, both original and projected as observed by the imaging spectrometer under evaluation.

\section{Spatial characterization}

Equally important for mapping applications, the spatial calibration and characterization of CIS cameras is critical to the ability of such systems to create products with the recoverable geometry and spatial fidelity needed to create cartographic products that meet mapping standards. This is especially critical for CIS systems as these sensors have small imaging arrays and are more sensitive to roll, pitch, and yaw perturbations when being operated from less stable lightweight platforms.

Internal CIS calibration procedures and reporting parameters, generally address the 'keystone effect' of camera systems. Sometimes also called the tombstone, or projective effect, it is the result of the camera platen not being perpendicular to the target surface and resulting in an image that is geometrically more similar to a trapezoid than a square. In imaging spectrometers, it is the measure of the spatial shift between pixels at different wavelengths, due to a change in optical magnification. It is an important source of spectral error, especially in push-broom sensors [41].

However, greater technical characterization is needed for systems intended for formal mapping purposes. USGS has a long history of providing calibration services for analog aerial cameras. The USGS Optical Sciences Lab (OSL) and the camera calibration reports they generate and process became a de facto standard in the aerial mapping field. With the advent of fully digital mapping cameras, the USGS decided it would not provide this same service for digital cameras, as USGS is not a formal legal certification authority. USGS continues, however, to provide research and assistance to the digital mapping process. For example, USGS has developed a 'Type Certification' for aerial sensors that certifies that any particular system, when used according to the manufacturer's specifications, has a high likelihood of consistently delivering high quality data.

Further, while earlier camera calibration standards focused on the photogrammetric process in establishing known errors from cameras, digital standards relate more to the quality of the final mapping products. This is accomplished by the use of a calibrated test range where known and precisely located targets can be used as quality control points for accurate geo-registration and ortho-rectification. Such calibrated test ranges have been proposed and implemented by USGS and exist in several locations across the country [21].

The USGS plan for quality assurance (QA) for digital aerial imagery and implementation plan $[42,43]$ outline two domains for a comprehensive approach to QA for the spatial accuracy component of digital aerial imagery: the data generation domain and the data procurement domain. These domains were primarily intended to address larger format mapping systems such as those utilized to create USGS and other federal map products. Most small CIS systems will likely not have the overall airframe stability or the sensor footprint sufficient to meet National Mapping Standards. However, the USGS Quality Assurance Plan for Digital Aerial Imagery does provide a comprehensive framework for assessing the spatial quality of a digital image product by creating dense, closely space geometric target at several test ranges, specifically designed for small aerial systems [23].

First, the data generation domain includes the development of a voluntary, cost-share, sensor type certification process which is an unbiased assessment of commercial aerial systems, similar to the traditional USGS role in camera calibration reports for analog mapping cameras. The USGS 2008 report [44] outlines detailed requirements and specifications for digital aerial sensor certification.

The remaining activities under the data generation domain involve a process called the data provider evaluation in which potential vendors fly imagery over USGS test ranges and provide defined ortho-mosaic products.

Second, the data procurement domain consists of two parts. First is the process of "...clearly, concisely, and quantifiably specifying what is desired in a business agreement..." for contracting aerial imagery and related mapping products. This includes templates for contracting mapping services that define terms and establishing procedures for the full mapping process, from data acquisition to the quality assurance required for final product acceptance.

The second part of the data procurement domain is the establishment of quality assessment standards and tools. To achieve 
this, the USGS has worked extensively with the Inter-Agency Digital Imagery Working Group (IADIWG) and together have established initial standard metrics and methods for measuring digital mapping imagery quality and acceptance.

While most of the USGS efforts are directed towards larger mapping cameras and probably not directly applicable to compact systems, this body of work can serve as a useful guide to the establishment of the proper set of spatial accuracy standards for CIS systems. More details on the USGS work on digital standards can be found at: http://calval.cr.usgs.gov/past-activities/digaerial/

\section{Discussion and Future Needs}

Hyperspectral technology, and particularly HSI sensors on UAVs, will be a major development for remote sensing technology. This technology is facilitating the ability of principal investigators to collect spectral imagery at high spatial resolution (i.e. sub $\mathrm{cm}$ ) and high frequency (i.e., several visits per day). This is in contrast to the satellite sensor imagery at $10 \mathrm{~s}$ of $\mathrm{m}$ spatial and typically 4-16 day revisit periods. The performance of the range of CIS poses a challenge to researchers who expect satellite sensor grade data products. Characterizing the sensor performance with recognized metric will help facilitate selecting the right sensor for the right task. In this expanding filed where demonstrations of hyperspectral capabilities greatly outnumber examples of established practices, calibrated sensors with traceability to an NMI such as NIST will foster a degree of confidence in areas that need reliable and repeatable results. As such, both the government and the user community share the responsibility to see that it is developed in a manner that expectations are clear and predictable. This paper represents an initial effort to that end. Finally, with the multitude of applications, it will likely necessitate the reconsideration of the application of standards in order to maintain flexibility in newly developed practices.

\section{Acknowledgements}

This research was funded by the USGS Climate and Land Use Mission area and the Land Remote Sensing Program, the National Institute of Standards and Technology, Sensor Sciences Division and the Mitre Corporation. Any use of trade, firm, or product names is for descriptive purposes only and does not imply endorsement by the U.S. Government. Special thanks to Bernard Hubbard of USGS for review, suggestions and editorial comments.

\section{References}

1. Gupta SG, Ghonge MM, Jawandhiya P (2013) Review of unmanned aircraft system (UAS) technology. IJARCET 2: 1646-1658.

2. Schaepman-Strub G, Schaepman ME, Painter TH, Dangel S, Martonchike JV (2006) Reflectance quantities in optical remote sensing-Definitions and case studies. Remote sensing of environment 103: 27-42.

3. Rao NR, Garg P, Ghosh S (2007) Development of an agricultural crops spectral library and classification of crops at cultivar level using hyperspectral data. Precision Agriculture 8: 173-185

4. Zhi L, David Zhang, Jing-qi Yan, Qing-Li Li, Qun-lin Tang (2007) Classification of hyperspectral medical tongue images for tongue diagnosis. Computerized Medical Imaging and Graphics 3: 672-678.

5. Martin ME, Wabuyele MB, Chen K, Kasili P, Panjehpour M, et al. (2006) Development of an advanced hyperspectral imaging ( $\mathrm{HSI}$ ) system with applications for cancer detection. Ann Biomed Eng 34: 1061-1068.

6. Lu G, B Fei (2014) Medical hyperspectral imaging: a review. J Biomed Opt 19: 010901.

7. Serranti S, Gargiulo A, Bonifazi G (2012) Hyperspectral imaging for process and quality control in recycling plants of polyolefin flakes. Journal of Near Infrared Spectroscopy 20: 573.

8. Tatzer P, Wolf M, Panner T (2005) Industrial application for inline material sorting using hyperspectral imaging in the NIR range. Real-Time Imaging 11: 99-107.

9. Gowen A, Colm O Donnel, Cullen P, Bell S (2008) Recent applications of chemical imaging to pharmaceutical process monitoring and quality control. European journal of pharmaceutics and biopharmaceutics 69: 10-22.
10. Gowen A, Colm O Donnel, Cullen P, Downey G, Frias JM (2007) Hyperspectral imaging-an emerging process analytical tool for food quality and safety control. Trends in Food Science \& Technology18: 590-598.

11. Lorente D, Aleixos N, Gomez-Sanchis J, Cubero S, Garcia-Navarrete O L, et al. (2012) Recent advances and applications of hyperspectral imaging for fruit and vegetable quality assessment. Food and Bioprocess Technology 5: 1121-1142.

12. Edelman G, Gaston E, van Leeuwen TG, Cullen PJ, Aalders MC (2012) Hyperspectral imaging for non-contact analysis of forensic traces. Forensic science international 223: 28-39.

13. Payne G, Langlois N, Lennard C, Roux C (2007) Applying visible hyperspectral (chemical) imaging to estimate the age of bruises. Med Sci Law 47: 225-232.

14. Randeberg LL, Larsen ELP, Svaasand LO (2010) Characterization of vascular structures and skin bruises using hyperspectral imaging, image analysis and diffusion theory. J Biophotonics 3: 53-65.

15. Thiemann S, Kaufmann $H$ (2002) Lake water quality monitoring using hyperspectral airborne data-a semiempirical multisensor and multitemporal approach for the Mecklenburg Lake District, Germany. Remote sensing of Environment 81: 228-237.

16. Anderson J, Reynolds C, Ringelberg D, Edwards J, Foley K (2008) Differentiation of live-viable versus dead bacterial endospores by calibrated hyperspectral reflectance microscopy. J Microsc 232: 130-136.

17. Pengra BW, Johnston CA, Loveland TR (2007) Mapping an invasive plant, Phragmites australis, in coastal wetlands using the EO-1 Hyperion hyperspectral sensor. Remote Sensing of Environment 108: 74-81.

18. Underwood E, Ustin S, DiPietro D (2003) Mapping nonnative plants using hyperspectral imagery. Remote Sensing of Environment 86: 150-161.

19. Hestir EL, Shruti Khannaa, Margaret E Andrewa, Maria J Santosa, Joshua H Viers (2008) Identification of invasive vegetation using hyperspectral remote sensing in the California Delta ecosystem. Remote Sensing of Environment 112: $4034-4047$

20. (2009) NIST, Mission, Vision, Core Competencies, and Core Values.

21. Christopherson J (2004) Camera and Sensor Calibration at USGS.

22. (2011) USGS, USGS Announces "No USGS Digital Camera Certification Requirement".

23. Sampatha A, Don Moe, Christopherson J, Stensaas G (2010) Geometric Evaluation and Validation of Aerial and Satellite Data using Sioux Falls Geometric Test Range. ASPRS Annual Conference San Diego, California.

24. Allen DW, George P Eppeldauer, Steven W Brown, Edward A Early, et al. (2003) Calibration and characterization of trap detector filter radiometers. SPIE 5151, USA

25. Early EA, Steven W Brown, David W Allen, Carol Johnson B, Brett C Bush (2002) Radiometric calibration of the Scripps Earth Polychromatic Imaging Camera. SPIE 4483, USA.

26. Kostkowski H J (1997) Reliable spectroradiometry. Spectroradiometry Consulting, the University of Michigan, USA.

27. Palmer JM, Grant BG (2010) The art of radiometry. SPIE Press Bellingham.

28. (2009) NIST, Primary optical watt radiometer (POWR).

29. (2009) NIST, Spectral irradiance and radiance responsivity calibrations using uniform sources (SIRCUS)

30. Eismann MT (2012) Hyperspectral remote sensing.SPIE Press Bellingham.

31. Schott JR (2007) Remote sensing: Oxford University Press.

32. Dadon A, Ben-Dor E, Karnieli A (2010) Use of derivative calculations and minimum noise fraction transform for detecting and correcting the spectral curvature effect (smile) in Hyperion images. IEEE Transactions on Geoscience and Remote Sensing 48: 2603-2612.

33. (2009) NIST, Hyperspectral Image Projector (HIP).

34. Rice JP, Steven W Brown, David W Allen, Howard W Yoon, Maritoni Litorja, Jeeseong C Hwang (2012) Hyperspectral image projector applications. SPIE 8254, USA.

35. Allen DW, Rice JP, Goodman JA (2009) Hyperspectral projection of a coral reef scene using the NIST hyperspectral image projector. SPIE 7334, USA.

36. Xu R X, David W Allen, Jiwei Huang, Surya Gnyawali, James Melvin, et al. (2012) Developing digital tissue phantoms for hyperspectral imaging of ischemic wounds. Biomedical Opt Express 3: 1433-1445.

37. Allen DW, Maxwell S, Rice JP, Chang R, Litorja M, et al. (2011) Hyperspectral image projection of a pig kidney for the evaluation of imagers used for oximetry. SPIE 7906, USA. 
38. Rice JP, Allen DW (2009) Hyperspectral image compressive projection algorithm. SPIE 7334, USA.

39. Allen DW, Ronald G Resmini, Christopher J Deloye, Jeffrey R Stevens (2013) A microscene approach to the evaluation of hyperspectral system leve performance. SPIE 8743, USA.

40. Cooksey CC, Tsai BK, Allen DW (2014) A collection and statistical analysis of skin reflectance signatures for inherent variability over the $250 \mathrm{~nm}$ to $2500 \mathrm{~nm}$ spectral range. International Society for Optics and Photonics.
41. (2012) SpecTIR, Glossary of Terms.

42. Stensaas G, Lee GY, Christopherson J, George YG Lee, Donald Moe, Robert Ryan (2006) The USGS plan for quality assurance of digital aerial imagery. MAPPS Conference, USA.

43. (2010) USGS, USGS Quality Assurance plan for Digital Aerial Imagery: Implementation Proposal.

44. (2008) USGS, USGS Quality assurance Plan for digital aerial imagery, Part 2-Type Certification of Sensor System. 